\title{
Design of Bandwidth Adjustment Receiver for Optical Wireless Communication
}

\author{
M. F. L. Abdullah \\ Department of Communication Engineering \\ Faculty of Electrical \& Electronic Engineering \\ University Tun Hussein Onn Malaysia \\ faiz@uthm.edu.my
}

\begin{abstract}
Infrared wireless communication possesses two main attractive advantages over its radio frequency counterpart, namely the abundance of unregulated spectrum in $700 \mathrm{~nm}-1500 \mathrm{~nm}$ region and the ease with which the IR radiation can be confined. Integrating microwave electronics and optics, it is possible to provide wideband communication services but it is well known that the signal level in an optical wireless receiver is weakest at the front end. This paper presents the concerned with a systematic approach to the design of receiver for indoor optical wireless communication. In particular, it is concerned with how one properly chooses the front-end preamplifier and biasing circuitry for the photodetector; and comparison of technique using bandwidth adjustment for better service quality with a bootstrapped transimpedance amplifier is presented. A controllable capacitance is introduced at the output of the second stage of the amplifier. This technique permits a bandwidth adjustment from $52 \mathrm{~Hz}$ to $233 \mathrm{MHz}$ for a capacitance range of $10 \mathrm{uF}$ to $1 \mathrm{pF}$.
\end{abstract}

Index Terms: photodetector, transimpedance preamplifier, bandwidth adjustment

\section{Introduction}

Wireless infrared transmission or optical wireless nowadays, has entered homes, offices, industry and health care, with applications in the field of remote control, telemetry and local communication. One of the prime motivators for considering the use of an optical carrier in the wireless context is the demand for greater transmission bandwidths. This is due the fact that radio frequency spectrum is already exceedingly congested and frequency allocations of sufficient bandwidths are extremely hard to obtain [1]. The intention of this paper is to provide insight into the research effort to date in optical wireless receiver both in academic and industrial contexts. The idea of using the optical medium for wireless communications is not new, having been proposed as a means for indoor communications almost two decades ago[2][3]. However, recent years have seen an increasing interest in the potential for free space optical systems to provide portable data communications. Proponents of optical wireless systems argue that the optical medium is the only cost-effective way to provide high bit-rate mobile services to volume markets. In most proposed infrared optical wireless communication [1-4], it is well known that the signal level in an optical wireless receiver is weakest at the front end. Weakest front end means that it is too noisy, too slow or both. The two parameters are not unrelated; it is easy to have a fast front end by preparing to sacrifice signal to noise ratio. This is where the system signal-to-noise ratio is determined and system performance level established.

\section{Theory of photodetector}

Semiconductor junctions that convert photon energy of light into an electrical signal by releasing and accelerating current-conducting carriers, ultimately to produce a baseband voltage for regeneration is called a photodiode[5]. In order to appreciate its performance in

Received: February $29^{\text {th }}, 2012$. Accepted: October $30^{\text {th }}, 2012$ 
practical optical wireless systems, we have to characterize the photodiode from three points of view: the physical viewpoint, the circuit viewpoint and the statistical viewpoint.

\section{A. Photodetector physical viewpoint}

The presence of incident optical power, entering a semiconductor device produces thermal agitation that release holes-electron pairs generated at various points within the diode as illustrated in Fig 1[6]. These carriers drift toward opposite ends of the device under the influence of the applied field. When a carrier passes through the high-field region, it may gain sufficient energy to generate one or more new pairs of holes and electrons through collision ionization. These new pairs will in turn generate additional pairs by the same mechanism. Carriers accumulate at opposite ends of the diode, thereby reducing the potential across the device until they are removed by the biasing and other circuitry in parallel with the diode as shown in Fig 2. The chances that a carrier will generate a new pair when passing through the high-field region depends upon the type of carrier, the material out of which the diode is constructed and the voltage across the device. The depth and extent of the junction determines the location of the depletion region and the light wavelengths that produce an efficient response. For a given photodiode and a given wavelength, a photodiode responsivity expresses the resulting efficiency through[6].

$$
\mathrm{I}_{\mathrm{p}}=\mathrm{r}_{\varnothing} \emptyset_{\mathrm{e}}
$$

where $\mathrm{r}_{\varnothing}$ - diode's flux responsivity and $\emptyset_{\mathrm{e}}$ - radiant flux energy in watts

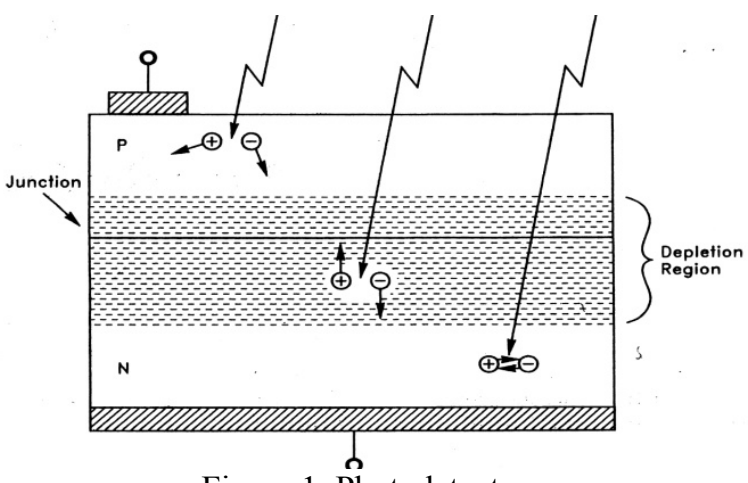

Figure 1. Photodetector

\section{B. Photodetetcor circuit viewpoint}

From the discussion above and more detailed investigation, modeling the characteristics of the photodiode with discreet circuit components permits analysis of application circuits. Fig 2 shows the resulting model with an ideal diode, a current source and parasitic elements. $\mathrm{C}_{\mathrm{d}}$ is the junction capacitance of the diode across which voltage accumulates when charges produced within the device separate under the influence of the bias field. The current generator $i_{p}(t)$ represents the production of charges by optical and thermal generation and collision ionization in the diode high-field region. Resistance $\mathrm{R}_{\mathrm{D}}$ represents the diode's dark resistance, which is the resistance of the zero-biased diode junction. In order to use the photodiode efficiently we must design a circuit which will respond to the current $i_{p}(t)$ with as little distortion and added noise as possible. In order to derive information from the circuit responding to $i_{p}(t)$ we must understand the statistical relationship between $\mathrm{i}_{\mathrm{p}}(\mathrm{t})$ (the equivalent current generator) and the incident optical power $\mathrm{p}(\mathrm{t})$ [5-6].

\section{Photodetector statistical viewpoint}

As shown in Figure 2, the current source $i_{p}(t)$ can be considered to be a sequence of impulses corresponding to electrons generated within the photodiode due to optical or thermal 
excitation or collision ionization. From various physical studies it has been concluded that for cases of current interest, the electron production process can be modeled as shown in Fig 3. Let the optical power falling upon the photon counter be $\mathrm{p}(\mathrm{t})$. In response to this power and due to thermal effects, the photon counter of Fig 3 produces electrons at average rate $\lambda(t)$ per second where

$$
\lambda(\mathrm{t})=[(\eta / \mathrm{h} \Omega) \mathrm{p}(\mathrm{t})]+\lambda_{\mathrm{o}}
$$

where $\eta$ - photon counter quantum efficiency

$$
\begin{aligned}
& \mathrm{h} \Omega \text { - energy pf a photon } \\
& \lambda_{\mathrm{o}}-\text { dark current "counts" per second }
\end{aligned}
$$

$\lambda(t)$ is only the average rate at which electrons are produced. $p(t)$ the number of electronic produced in any interval is statistically independent of the number produced in any other disjoint interval.[5]

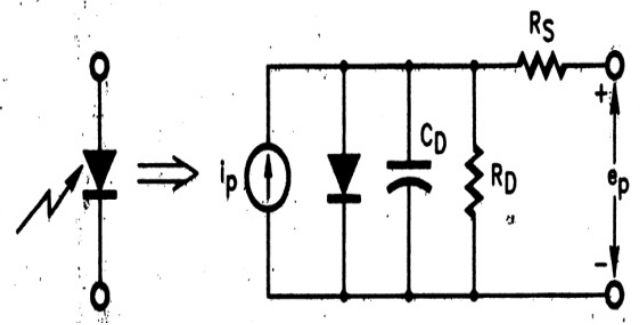

Figure 2. Photodiode circuit model
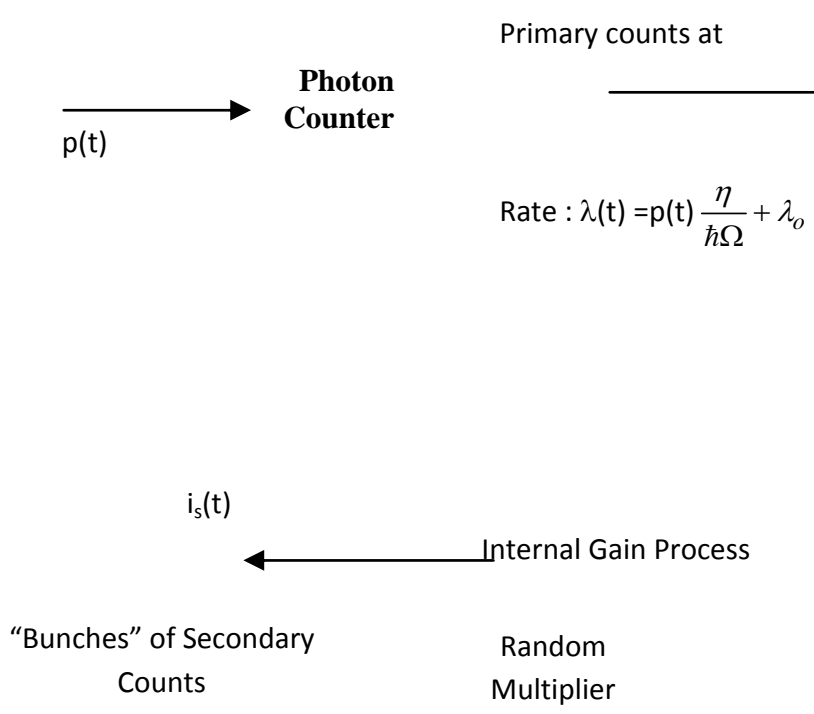

Figure 3. Model of $i_{s}(t)$ generation process

Each of the primary impulses produced by the photon counter enters a random multiplier where, corresponding to collision ionization it is replaced by g contiguous "secondary" impulses. The number $\mathrm{g}$ is governed by the statistics of the internal gain mechanism of the photodiode. Each primary impulse is "multiplied" in this manner by a value $g$ which is 
statistically independent of the value $g$ assigned to other primaries. Thus the current leaving the photodiode consists of bunches of electrons. For applications interest here, it will be assumed that all electrons in a bunch exit the photodiode at the time when the primary is produced. This shows that the duration of the photodiode response to a single primary hole-electron pair is very short compared to the response times of circuitry to be used with the photodiode.

\section{Types of Photodetector}

Photodetector consists of two variations of the basic photodiode that improves the diode's response. Physical study shows that PIN photodiodes increase the spectral bandwidth or range of light frequencies that produce an efficient photo response. Avalanche photodiodes increase the magnitude of the output current and the response speed by permitting diode bias at the verge of breakdown. For application interest, PIN photodiode is preferably in optical wireless communication system.

In terms of Noise Contributions: As a photodiode amplifier, the current to voltage converter exhibits a complex noise behavior. The major sources of noise in front ends are listed in Table 1 , where $e_{N}$ and $i_{N}$ are rms values of random fluctuations [4],[7].

Table 1. Noise source

\begin{tabular}{|l|l|l|l|}
\hline Source & Type & Formula & Dominates When : \\
\hline Photocurrent & Shot noise & $\mathrm{i}_{\mathrm{NSHOT}}=\left(2 \mathrm{eI}_{\mathrm{d}}\right)^{1 / 2}$ & Bright light, large load resistor \\
\hline Load resistor & Johnson noise & $\mathrm{I}_{\mathrm{Nth}}=(4 \mathrm{kT} / \mathrm{R})^{1 / 2}$ & Dim light, small R \\
\hline Amplifier & Input current noise & $\mathrm{i}_{\mathrm{N}}=\sqrt{\frac{4 k T}{R}}$ & Ideally, never \\
\cline { 2 - 4 } & $\begin{array}{l}\text { Input voltage } \\
\text { noise }\end{array}$ & $\mathrm{e}_{\mathrm{N}}=\sqrt{4 k T R}$ & $\begin{array}{l}\text { Dim light, large RC or a fast } \\
\text { noisy amp }\end{array}$ \\
\hline
\end{tabular}

\section{Receiver Preamplifier Structures}

This section is an example of how to choose and design a front-end amplifier for a visible or near IR photodiode and how to get improvement in bandwidth without a big sacrifice of SNR. The receiver preamplifier performs the critical function of interfacing the photodiode to the rest of the receiver. Typically, the preamplifier converts the received photocurrent into a voltage signal. The preamplifier plays a crucial role in determining many aspects of the overall performance of the receiver including speed, sensitivity, and dynamic range. Receiver preamplifiers are typically classified in categories as either a High-impedance amplifier or a $\mathrm{T}$ trans impedance amplifier.

High impedance amplifier theory: Figure 4 shows a typical receiver in schematic form consisting of a photodiode, an amplifier and an equalizer. This amplifier is modeled as an ideal high-gain impedance amplifier with an equivalent shunt capacitance and resistance at the input, in addition with two noise sources referred to the input. The noise sources for this particular part will be assumed to be white, Gaussian and uncorrelated. It is also assumed that amplifier gain is sufficiently high, that the noises introduced by the equalizer are negligible. The average detector output current, $i_{S}(t)$ is given by $:[5]$

$$
\mathrm{i}_{\mathrm{S}}(\mathrm{t})=\frac{\eta e p(t)}{\hbar \Omega}+e \lambda_{o}
$$

where $\mathrm{e}=$ electron charge, $\lambda_{\mathrm{o}}=$ dark current electrons/second, $\frac{\eta p(t)}{\hbar \Omega}=$ average optical primary electrons/second.

Therefore the average voltage (neglecting dc components) at equalizer output, where “* * indicates convolution and $\mathrm{A}$ is an arbitrary constant : 
$\mathrm{v}_{\text {out }}(\mathrm{t})=\frac{A \eta e p(t)}{\hbar \Omega} * h_{f e}(t) * h_{e q}(t)$
$\mathrm{h}_{\mathrm{fe}}(\mathrm{t})=\mathrm{F}\left\{\frac{1}{\frac{1}{R_{T}}+j \omega\left(C_{d}+C_{A}\right)}\right\}$ is the amplifier input circuit current impulse response $\mathrm{R}_{\mathrm{T}}=$ $\left[\frac{1}{R_{b}}+\frac{1}{R_{A}}\right]^{-1}$ is the total detector parallel load resistance $\mathrm{h}_{\mathrm{eq}}(\mathrm{t})$ is the equalizer impulse response.

High impedance amplifier tends to give a degraded frequency response as the bandwidth relationship $\frac{1}{2 \pi R_{b}\left(C_{d}+C_{A}\right)} \geq B$ is not maintained for wideband operation [5]. The detector output is effectively integrated over a large time constant and must be restored by differentiation. This is performed with the correct equalization. Therefore the high impedance front end gives a better improvement in sensitivity, but eventually creates a heavy demand for equalization and has problem of limited dynamic range. The limited dynamic range is because of the attenuation from the low frequency signal components by the equalization process which causes the amplifier to saturate at high level signals. If the amplifier is saturated before equalization has occurred the signal will be heavily distorted, thus reducing the dynamic range which is dependent upon the amount of integration and subsequent equalization employed.

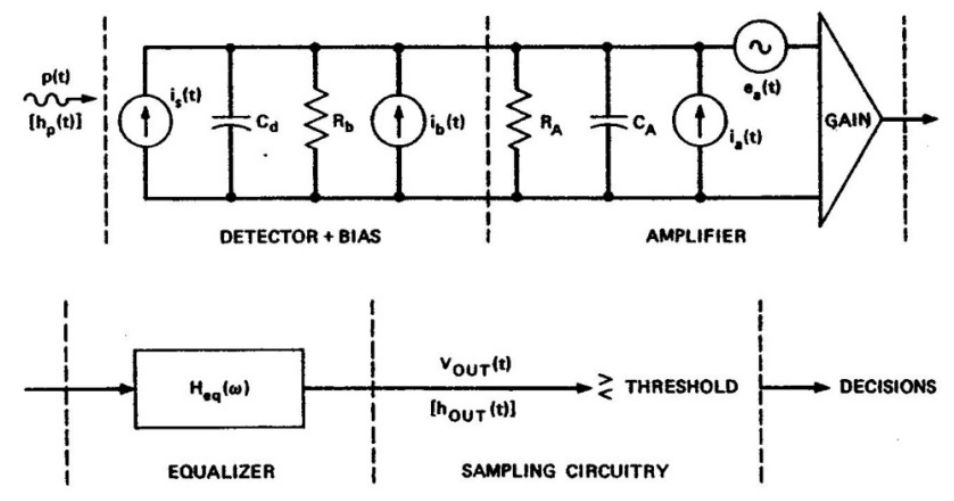

Figure 4. High impedance amplifier with equalizer

Receiver preamplifiers based on the transimpedance amplifier are currently the most popular because they avoid the dynamic range problem associated with high-impedance designs, and because they provide a good compromise between the wide bandwidth of the lowimpedance design and the low noise characteristics of the high-impedance design. Given a detector (Fig 2) whose output is a current, the easiest way to form a voltage from it is to have a load resistor $\left(R_{L}\right)$. The output full swing appears across the detector capacitance $C_{d}$, rolls off starting at $\mathrm{f}_{\mathrm{RC}}=\frac{1}{2 \pi R_{L} C_{D}}$. The signal voltage $\mathrm{V}_{\mathrm{o}}(\mathrm{f})=\frac{i_{p}(f) R_{L}}{1+j 2 \pi R_{L} C_{D} f}$. Reducing $\mathrm{R}_{\mathrm{L}}$ will reduce the $\mathrm{RC}$ product and speed up the system, while increasing $\mathrm{R}_{\mathrm{L}}$ increase the bandwidth and dynamic range of the system. Another key idea is to reduce the swing across $C_{D}$, by making the detector work into a virtual ground using a transimpedance amplifier shown in Fig 5(a) [4],[7]. The inverting input of $\mathrm{A}_{1}$ draws no current, feedback forces the voltage there to be close to zero at all times. $A_{1}$ senses the voltage across $C_{D}$ and wiggles other end of $R_{f}$ to zero it out. Provided that $A_{1}$ has high loop gain $A_{V O L}$, the swing across $C_{D}$ is greatly reduced and the bandwidth greatly improved. The amplifier input adds a significant amount of its own 
capacitance $\mathrm{C}_{\text {in }}$. For a typical transimpedance topology using active devices with load feedback resistance $\mathrm{R}_{\mathrm{f}}$, the transimpedance gain $\mathrm{A}_{\mathrm{z}}$ can be approximated by :

$$
\mathrm{A}_{\mathrm{z}} \approx \frac{-R_{f}}{1+j \omega \frac{R_{f}\left(C_{D}+C_{i n}\right)}{A_{V O}}}
$$

where $A_{v o}$ is the open loop voltage gain of the amplifier and $\omega$ is the angular frequency.

The transimpedance rolls off depends on the magnitude of the impedance of the feedback elements. Therefore the transimpedance amplifier bandwidth is calculated with the following equation :[4],[7]

$$
\mathrm{f}_{3 \mathrm{~dB}} \approx \sqrt{\frac{f_{R C} f_{T}}{2}}
$$

where $\mathrm{f}_{\mathrm{T}}$ is the unity-gain crossover frequency

Equation 6 shows that the upper $3 \mathrm{~dB}$ cut-off frequency of the preamplifier is a function of the capacitance from the detector, feedback resistor and open loop voltage gain. In other words, a large detector means a large $C_{d}$. Hence, in order to achieve large bandwidths either the value of $R_{f}$ is reduced or $A_{v o}$ is increased. Unfortunately, increasing $A_{v o}$ will jeopardize amplifier stability and reducing $R_{f}$ will increase thermal noise into the system.

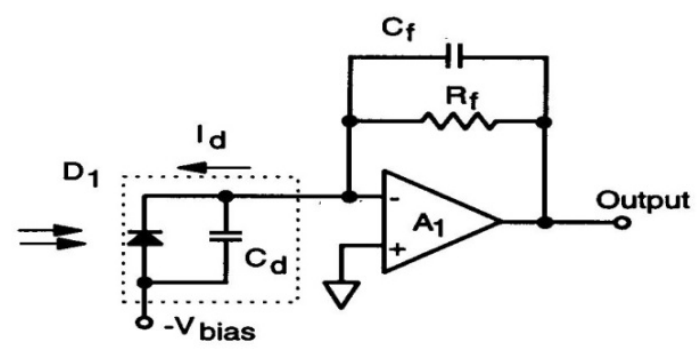

Figure 5(a). Transimpedance amplifier circuit

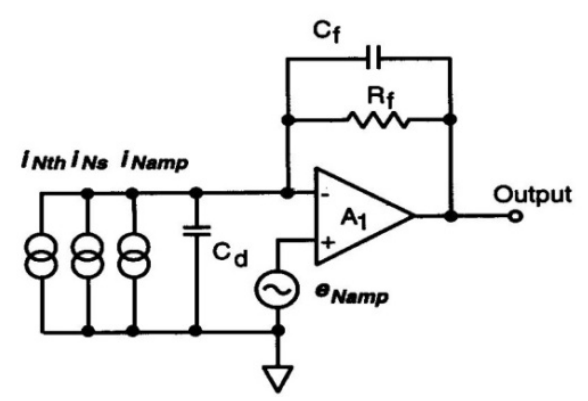

Figure 5(b). Transimpedance amplifier noise model

Noise in transimpedance amplifier : From Fig 5(b), it is obvious that all the current sources are in parallel $: i_{d}, i_{\text {NSHOT }}, i_{\text {Nth }}$ and $i_{\text {amp }}$. The Johnson noise $i_{\text {Nth }}$ appears across $R_{f}$ but since the op amp output impedance is low and the currents add linearly, the other end of $i_{\text {Nth }}$ is at ground for noise purpose. The rolloff in the frequency response does not degrade the signal to current noise ratio. The amplifier's voltage noise, $e_{N a m p}$ is treated differently because $A_{1}$ is a differential amplifier. Therefore $\mathrm{e}_{\mathrm{Namp}}$ is multiplied by $\mathrm{A}_{1}$ 's noninverting gain [4],[7]. 


$$
\mathrm{A}_{\mathrm{Vcl}}=\frac{A_{\mathrm{Vol}}}{1+\frac{A_{\mathrm{Vol}}}{1+j w C_{D} R_{f}}}
$$

If $\mathrm{e}_{\mathrm{Namp}}$ is very low or if we are not trying to get a huge bandwidth improvement through $\left(\mathrm{f}_{\mathrm{T}} * \mathrm{f}_{\mathrm{RC}}\right)^{1 / 2}$ mechanism, this rising noise contributions will not limit. Otherwise, it will dominate the noise starting at:

$$
\mathrm{f}_{3 \mathrm{~dB}}=\frac{1}{2 \pi e_{N a m p} C_{D}} \sqrt{2 e I_{D}+i_{N a m p}^{2}+\frac{4 k T}{R_{L}}}
$$

In order that op amp do not dominate the noise according to Hobbs[7], we should choose it by the following rules :

$\mathrm{i}_{\text {Namp }}<0.5 \mathrm{i}_{\mathrm{Nth}}$ (Noise of $\mathrm{R}_{\mathrm{f}}$ dominates $\mathrm{i}_{\mathrm{Namp}}$ )

$\mathrm{e}_{\mathrm{Namp}}<0.5 \mathrm{R}_{\mathrm{f}} \mathrm{i}_{\mathrm{Nth}}\left(\mathrm{e}_{\mathrm{Namp}}\right.$ should be the same in flatband)

$\mathrm{e}_{\mathrm{Namp}}<0.5 \mathrm{i}_{\mathrm{Nth}} /\left(2 \pi \mathrm{f}_{3 \mathrm{~dB}}\left(\mathrm{C}_{\mathrm{D}}+\mathrm{C}_{\mathrm{in}}\right)\right.$ (Noise peak should not dominate anywhere in the frequency band)

$\mathrm{f}_{\mathrm{T}}>2 \mathrm{f}_{3 \mathrm{~dB}}{ }^{2} / \mathrm{f}_{\mathrm{RC}}$ (The amplifier has to raise enough bandwidth)

$\mathrm{f}_{\mathrm{T}}<10 \mathrm{f}_{3 \mathrm{~dB}} 2 / \mathrm{f}_{\mathrm{RC}}$ (Speed too fast risks trouble with ringing and oscillation)

The transimpedance amplifier does not improve the SNR of the photodiode but it just changes the frequency response. There's nothing inherent or inescapable about noise peak in system, it comes from a poor choice of circuit topology that can be amended.

\section{Receiver Design}

A. Bandwidth enhancement technique

The majority of optical wireless receivers proposed [1][3][8][9][10][11] or demonstrated to date have employed transimpedance amplifier, due to the fact this configuration largely overcomes the drawbacks of high impedance front end by utilizing a low noise, high input impedance amplifier with positive or negative feedback. Bandwidth enhancement techniques are required that do not affect low frequency behavior, so that both high dc gain and large bandwidth can be obtained. Several of these techniques have been proposed in the literature in order to maximize the gain-bandwidth product of an amplifier stage.

One of the method is a transimpedance amplifier is built up with two capacitive coupled voltage dividers $\left(R_{1}-R_{2}, R_{3}-R_{4}\right)$ instead of a single feedback resistor[10]. The basic concept of this network is its different behavior at low and high frequencies. At low frequencies the transimpedance of the transimpedance amplifier is approximately the sum of $R_{1}$ and $R_{2}$. For higher frequencies the parasitic capacitance of resistor $R_{2}$ has no effect owing to the low resistance of $R_{4}$, which has no effect on the bandwidth of the transimpedance amplifier. Only parasitic capacitance of resistor $\mathrm{R}_{1}$ has an influence on the bandwidth of the circuit at a nine times higher frequency than before. Cascading and capacitance neutralization compensate the input Miller capacitance thus expanding the bandwidth if the input pole is dominant. Unfortunately these techniques are less effective in low power amplifiers that use high speed bipolar transistors, if the output pole becomes dominant.

Another well known method to increase bandwidth of amplifiers uses peaking capacitors[9] or inductors. This method usually places inductors or capacitors in a strategic location of the amplifier circuit, resulting in a resonance with parasitic capacitances, which broadens the bandwidth of the amplifier. Although inductive peaking do increase the amplifier bandwidth, stray capacitances of the inductor often causes bandwidth degradation rather than an improvement. Capacitive peaking design is preferable, but this technique is extremely sensitive to process variations and could cause large peaking. 
The topology being known as the bootstrap transimpedance amplifier (BTA) is an attractive design because it involves a positive feedback loop, which causes a point in the circuit to be pulled up as if by its own bootstraps. This principle is often used in high input impedance amplifiers, wide bandwidth designs, and in reducing the effective detector capacitance, $\mathrm{C}_{\mathrm{d}}$ from the photodiode as seen by the signal as discussed in [11][12][13][14]. Therefore, a much improved version of a front-end, incorporated within a transimpedance amplifier, in terms of bandwidth is shown in Fig 6 [15]. The output of the emitter follower stage is feedback to the photodetector by a bootstrapping capacitor, $\mathrm{C}_{3}$. Fig 7 proposes a combination of bootstrapped technique with a capacitive peaking technique by placing a capacitor, $\mathrm{C}_{4}$, in series with the emitter resistor in the second gain stage, with a feedback resistor $R_{8}$ [16]. By varying the capacitor, the bandwidth of the circuit can be controlled. The output results of Fig 6 and Fig 7 are shown in Fig 8 and Fig 9. In each case the amplifier output is taken from the collector at $\mathrm{Q}_{3}$. Assuming that the gain stages and the emitter follower can be approximated by a simplified hybrid- $\pi$ model, as shown in Figure $10, R_{1}, R_{2}>>R_{7}$ and we consider frequencies where $\mathrm{C}_{1}$, $\mathrm{C}_{2}$ and $\mathrm{C}_{3}$ are short circuits, the transimpedance gain, $\mathrm{A}_{\mathrm{z}}$ for the circuit is approximated by equation (9):

$$
\mathrm{A}_{\mathrm{z}}=\frac{V_{o}}{I_{p}}=\frac{g_{m 3} R_{6}\left[2 r_{3}+R_{7}(1-A)\right]}{(A+1)\left[1+r_{3} g_{m 3}\right]+j \omega C_{4}\left[r_{3}\left(1+g_{m 3} R_{7}\right)+R_{7}\right]}
$$

where $\mathrm{A}$ is the voltage gain of the first stage amplifier

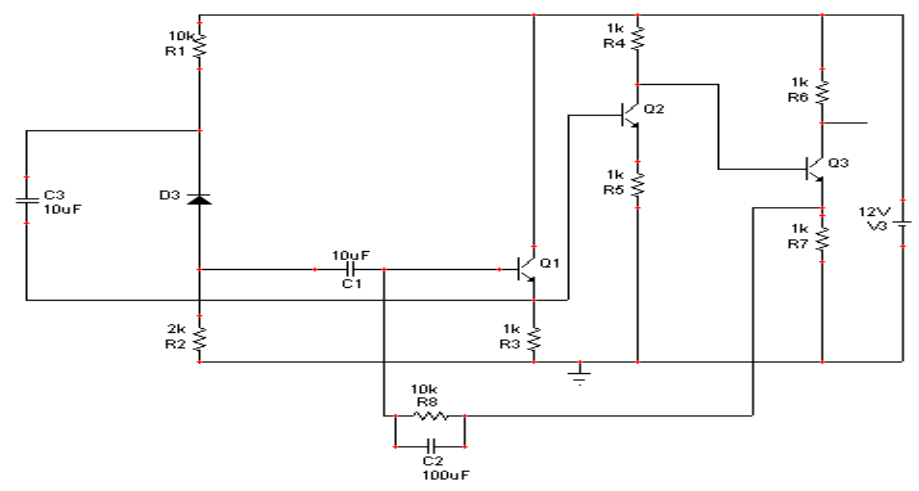

Figure 6. Bootstrapped transimpedance amplifier circuit

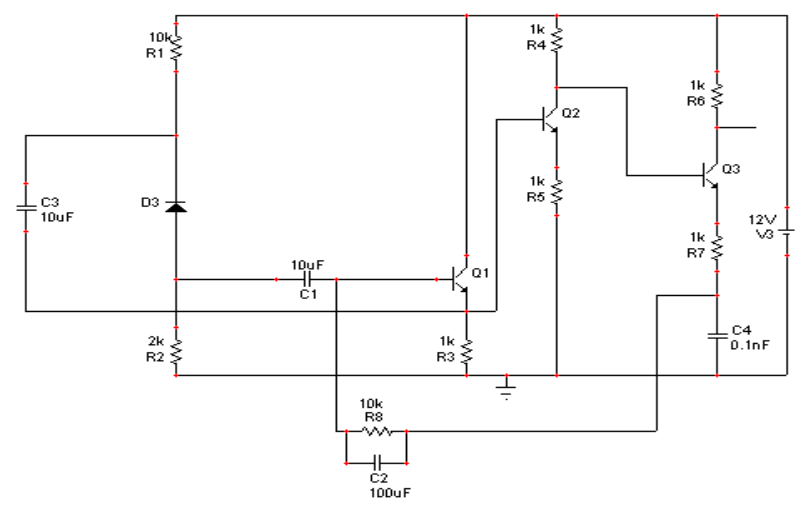

Figure 7. Bootstrapped with capacitor peaking transimpedance amplifier circuit 
Equation (9) shows that the receiver bandwidth is determined not only by the $\mathrm{R}_{8} \mathrm{C}_{\mathrm{d}}$ time constant but by a complex function of $g_{m 3}, r_{3}, R_{7}, R_{6}$ and $C_{4}$. The circuit effectively operates in between the low and the high frequency range. Thus, the modified circuit shows that varying capacitor $\mathrm{C} 4$, thus modifying the second stage gain can vary the bandwidth. The lower the value of $\mathrm{C}_{4}$, the higher the bandwidth becomes. This technique permits a bandwidth adjustment from $233 \mathrm{MHz}$ to $52 \mathrm{MHz}$ for a capacitance range of $1 \mathrm{pF}$ to $10 \mu \mathrm{F}$. The BTA circuit has a gain of $41 \mathrm{~dB}$ with a cut-off frequency of $1 \mathrm{GHz}$, while the modified BTA circuit maintains a gain of $48.2 \mathrm{~dB}$ as $\mathrm{C}_{\mathrm{p}}$ is varies until $\mathrm{C}_{\mathrm{p}}$ is $1 \mathrm{uF}$ and $10 \mathrm{uF}$, the gain starts to drop to $47.7 \mathrm{~dB}$ and $44.7 \mathrm{~dB}$ respectively. The stimulation shows that the bandwidth adjustment between each capacitor value is a ratio of $10: 1$ to $8: 1$.

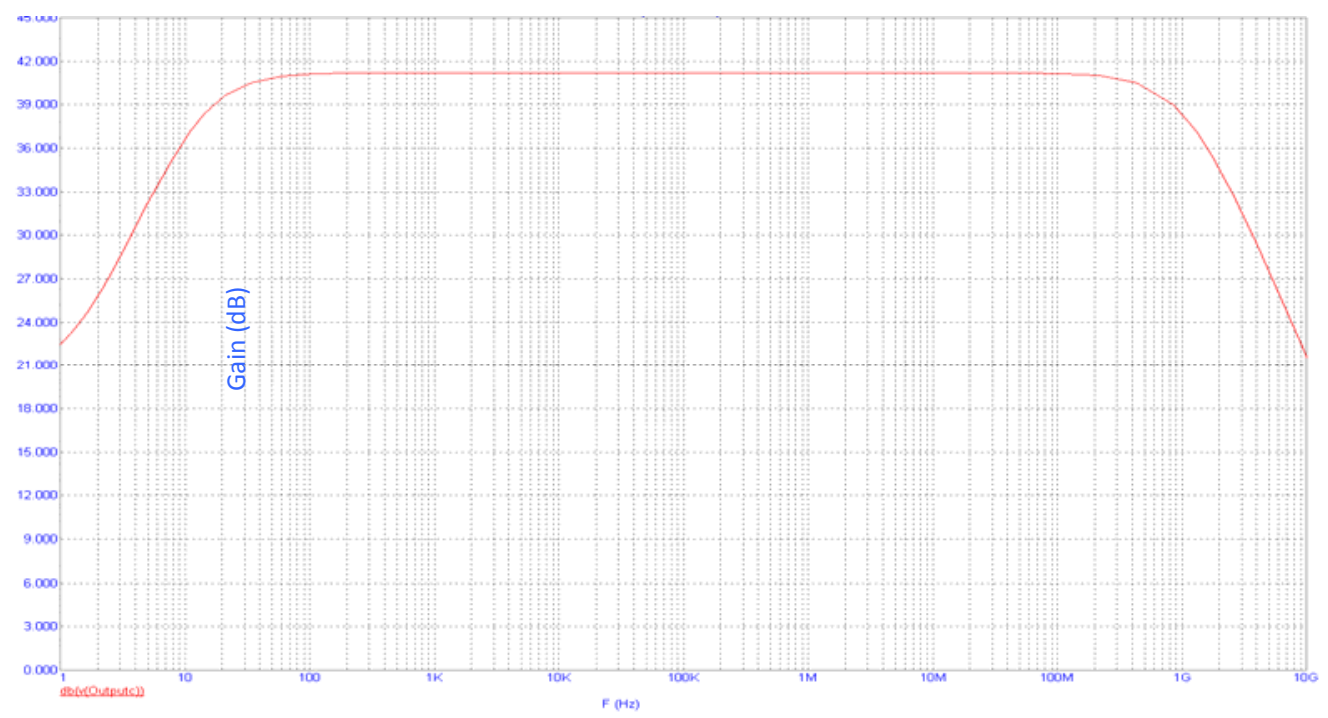

Figure 8. Frequency response bootstrapped transimpedance amplifier

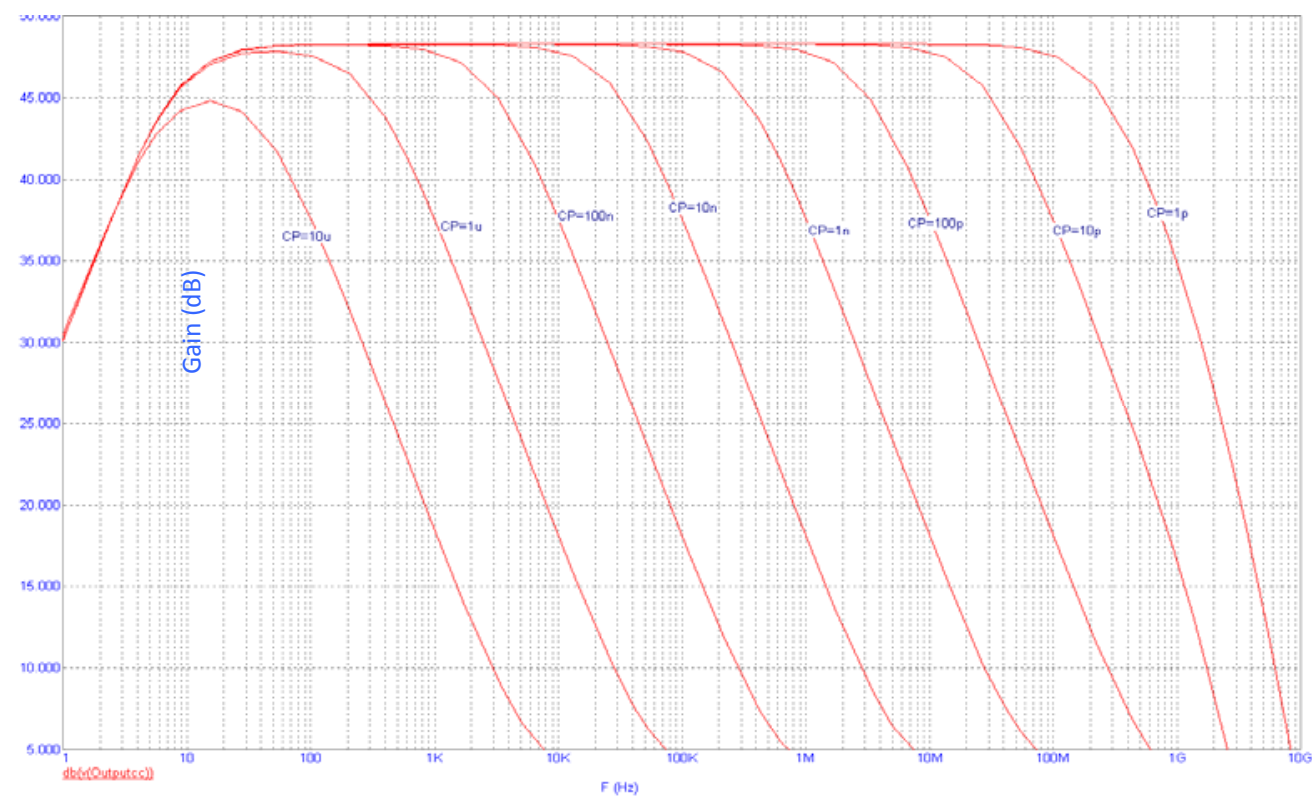

Figure 9. Frequency response bootstrapped with capacitor peaking transimpedance amplifier 


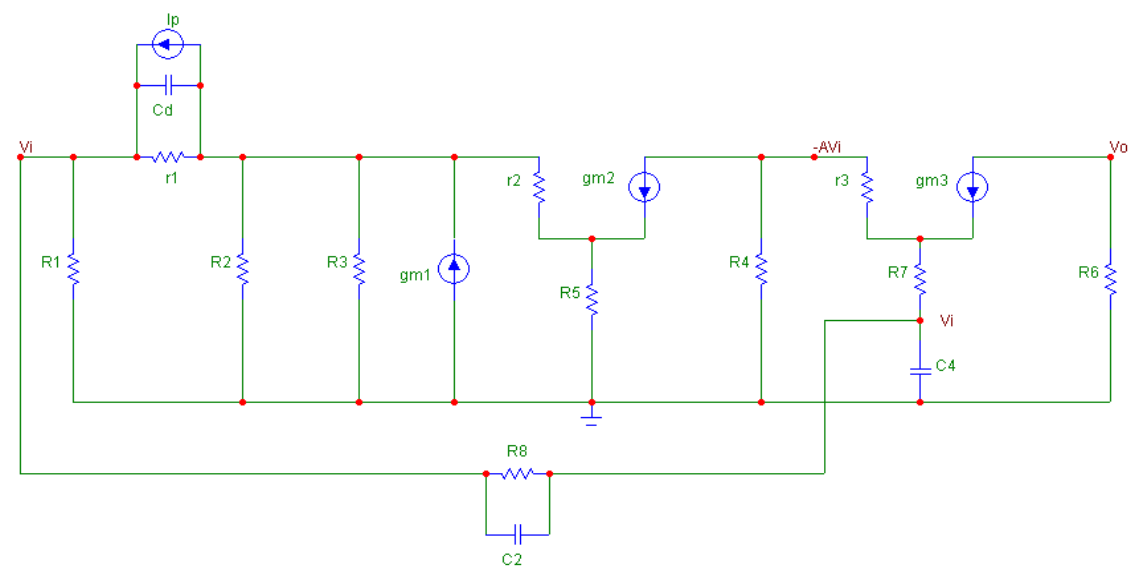

Figure 10. Model hybrid-pi of bootstrapped with capacitor peaking transimpedance amplifier circuit

A better proposed topology using a composite amplifier provides high bandwidth as shown in Fig 11. The bootstrapped transimpedance amplifier is connected in series with a voltage feedback amplifier and a RC filter. By varying the capacitor, $\mathrm{C}_{6}$ between $50 \mathrm{pF}$ to $1 \mathrm{nF}$ the bandwidth of the circuit can be controlled in the frequency range of $6 \mathrm{MHz}$ to $60 \mathrm{MHz}$ as shown in Fig 12. There is a trade-off between gain and bandwidth compared to Fig 9. As the bandwidth is increased the gain of the circuit is reduced. If we assume that the gain stages, the emitter follower and second stage amplifier can be approximated by a simplified hybrid- $\pi$ model, as shown in Figure 13. The transimpedance gain, $\mathrm{A}_{z 1}$ for the circuit can be approximated by equation (10) considering frequencies where $\mathrm{C}_{1}, \mathrm{C}_{2}$ and $\mathrm{C}_{3}$ are short circuits:

$$
\mathrm{A}_{\mathrm{z} 1}=\frac{V_{\text {o2 }}}{I_{p}}=\frac{R_{19} R_{i 1} R_{6} A_{1}\left[R_{7}+A R_{7} R_{6}-r_{3}\right]}{r_{3}\left(R_{19} R_{i 1}+R_{19}+A_{1} R_{i 1}\right)\left(1+j \omega C_{6} R_{20}\right)}
$$

where $A$ is the voltage gain of the first stage amplifier and $A_{1}$ is the voltage gain of the second stage amplifier.

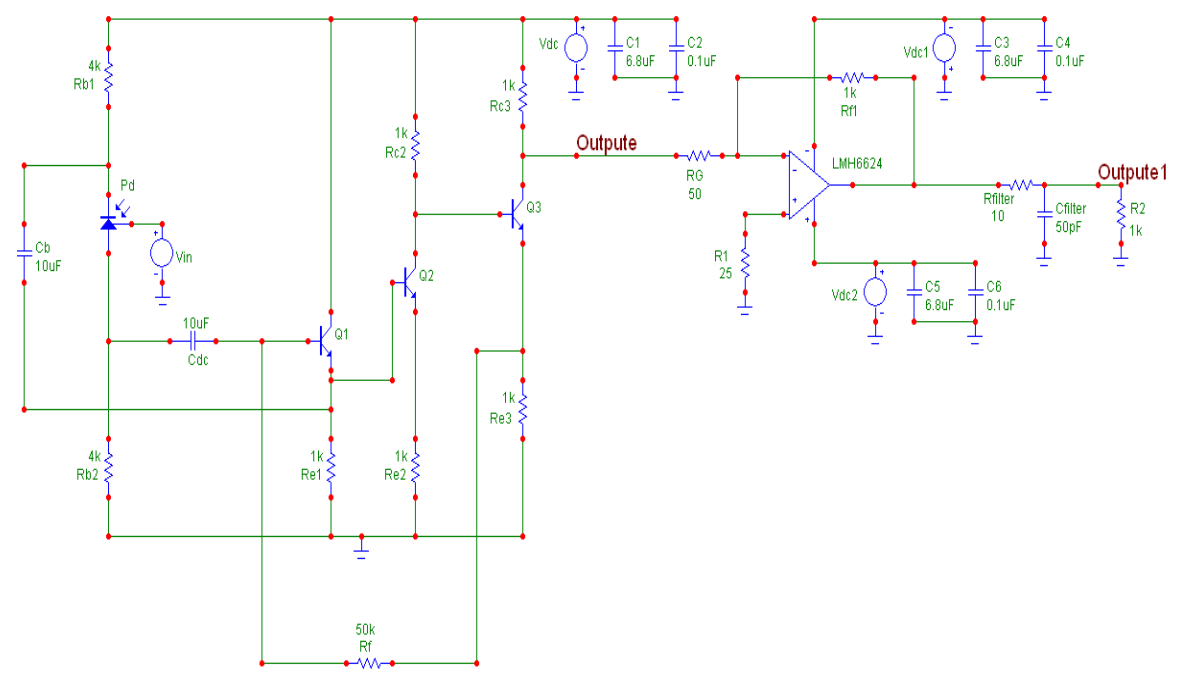

Figure 11. Composite transimpedance amplifier 
Bootstrapping transimpedance amplifier effectively allows for a higher transimpedance gain and a lower $\mathrm{R}_{\mathrm{f}}$ thermal noise contribution. Composite configuration of transimpedance amplifier effectively allows for a higher bandwidth with a trade-off of $10 \mathrm{~dB}-20 \mathrm{~dB}$ gain, while maintain its noise contribution. In application wise, it is suggest to use the composite amplifier receiver to accommodate the high frequency range bandwidth adjustment, where a variable switching capacitor circuit would be use to vary the capacitance from the range of $50 \mathrm{pF}$ to $1 \mathrm{nF}$. The propose switching circuit which is design to be incorporated together with the receiver will automatically select the right capacitance value based on the received signal quality and adjust the receiver to the required bandwidth.
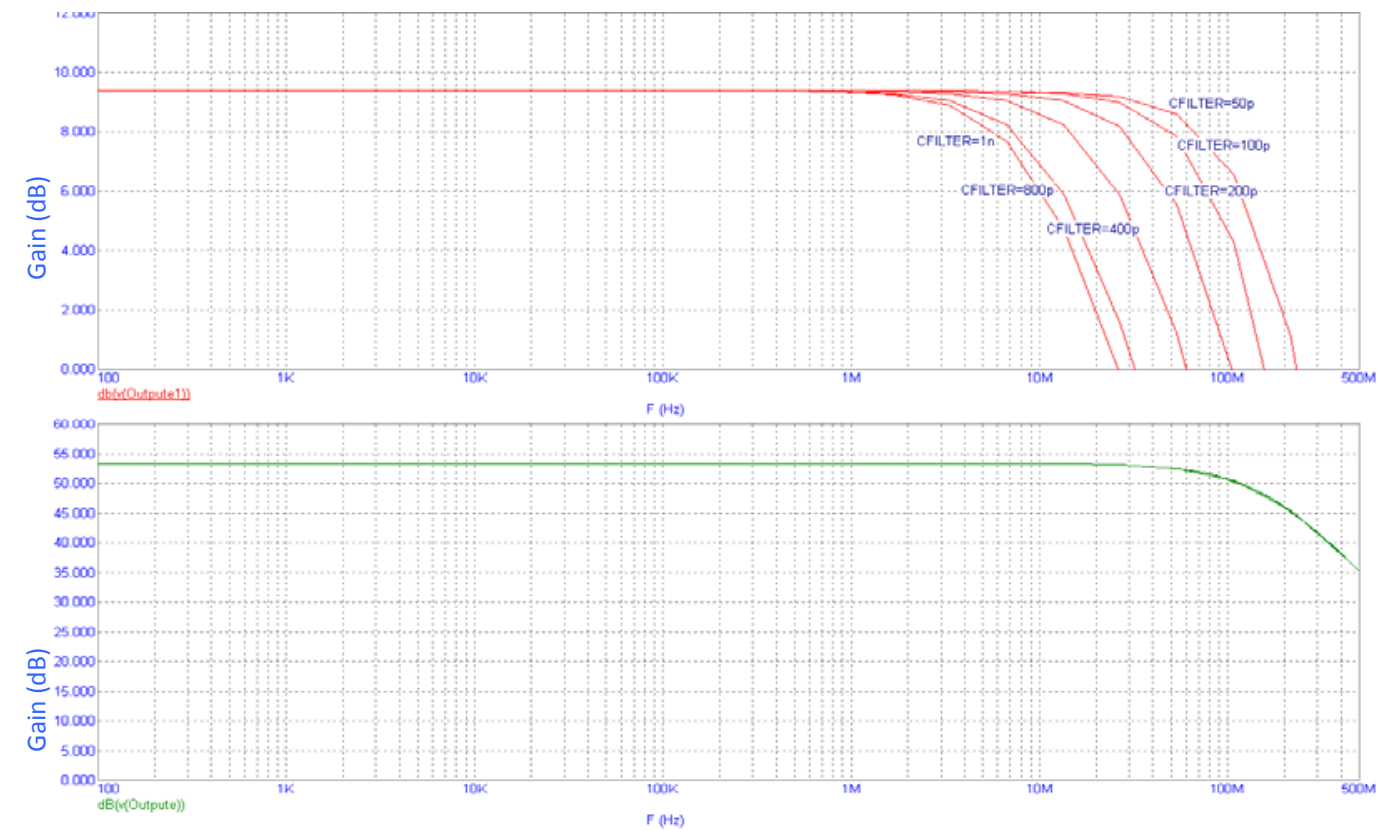

Figure 12. Frequency response composite transimpedance amplifier

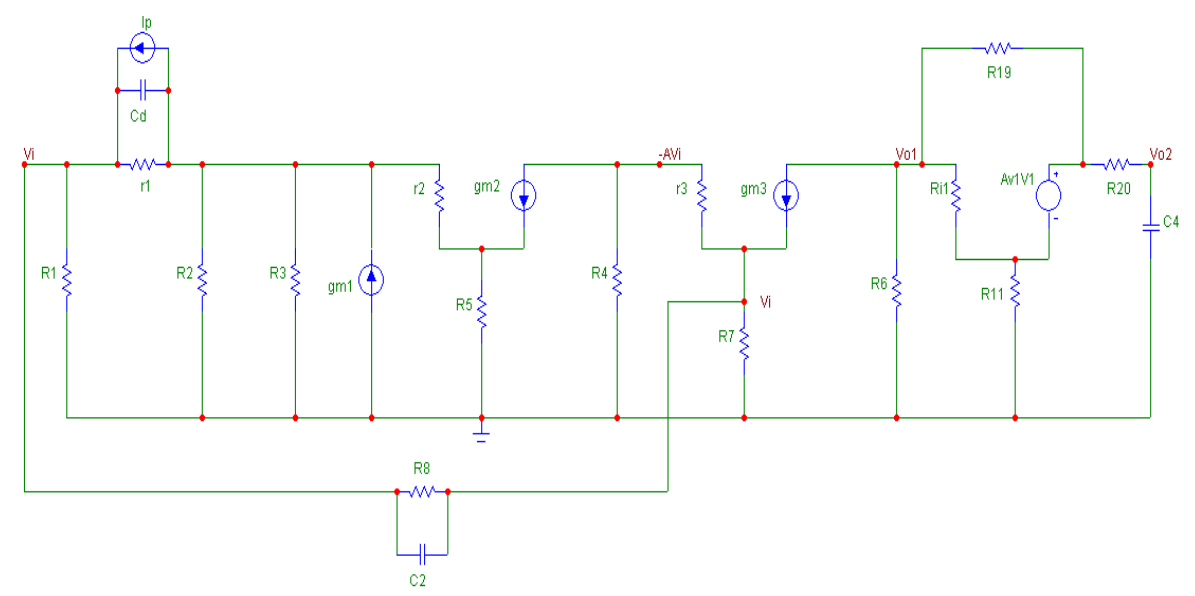

Figure 13. Model hybrid-pi of composite transimpedance amplifier 


\section{Conclusion}

Infrared wireless had yet to exploit fully all the potential benefits offered by the medium. There is still a great deal of work to be done in the adaptation and optimization of coverage areas, especially with unknown receiver orientation. Receiver design is particularly challenging because not only dynamic range and bandwidth criteria has to be met, but also significant problems of high ambient light levels noise has to be dealt with. This paper has provided an insight issues associated with the front-end design of a wireless infrared communication. It has highlighted the significant maxims on choosing the biasing circuitry. To summarize, high impedance amplifier with a large $\mathrm{R}_{\mathrm{f}}$ diminish the effects of thermal noise. However the receiver bandwidth is then usually smaller then the signal bandwidth, which require an equalization stage following the preamplifier as discussed. The circuit will be tricky, as the equalizer effect reduces the overall dynamic range of the receiver. The transimpedance front ends resolve these issues by using a large feedback resistor and an inverting amplifier, which boots the bandwidth without thermal noise and dynamic range problems. This paper also presented the bootstrap transimpedance amplifier technique using the adjustable capacitor has a wide bandwidth of frequency range, with an average gain of $48 \mathrm{~dB}$. The technique using bootstrap a transimpedance amplifier with a VFA as a composite amplifier has the lowest gain, $12.3 \mathrm{~dB}$, and the bandwidth adjustment is more focused in the centre between the high frequency range (HF) to very high frequency range (VHF). The bandwidth adjustment ratio for composite amplifier is much better as shown in Table 1.

Table 1. Comparison of BTA and BTA composite amplifier technique

\begin{tabular}{|l|c|c|}
\hline Technique & $\begin{array}{c}\text { Adjustable } \\
\text { capacitor }\end{array}$ & $\begin{array}{c}\text { Bootstrap TIA and } \\
\text { VFA }\end{array}$ \\
\hline Gain & $48.2 \mathrm{~dB}$ & $12.3 \mathrm{~dB}$ \\
\hline Cut-off & $52 \mathrm{~Hz}-233 \mathrm{MHz}$ & $9.5 \mathrm{MHz}-$ \\
Frequency & & $103.5 \mathrm{MHz}$ \\
\hline \multirow{2}{*}{ Ratio } & $1: 10$ (between & $1: 10.8$ \\
\hline
\end{tabular}

\section{References}

[1] A.M.Street, P.N.Stavrinou, D.J.Edwards and G.Parry, “ Optical Preamplifier designs for IR-LAN Applications" IEE (1996), pp : 8/1-8/6

[2] John R.Barry, "Wireless Infrared Communications" Kluwer Academic Publishers, 1994

[3] A.M.Street, P.N.Stavrinou, D.C.O'Brien and D.J.Edwards, “ Indoor optical wireless systems - a review" Optical and Quantum Electronics 29 (1997), pp : 349-378

[4] Stephen B.Alexander, "Optical Communication Receiver Design" IEE Telecommunications Series, Vol 37 and SPIE Tutorial Texts in Optical Engineering, Vol TT22, 1997

[5] S.D. Personick, "Receiver Design for Digital Fiber Optic Communication Systems I" The Bell System Technical Journal, Vol : 52, (1973), pp : 843 - 874.

[6] Graeme, J. G, "Photodiode amplifiers : Op amp solutions", McGraw Hill (1985).

[7] Philips C.D. Hobbs, "Building Electro-Optical Systems : Making it All Work", John Wiley \& Sons Inc, (2000), pp : $620-666$

[8] R.J. Green, M.G. Mcneill, "Bootstrap transimpedance amplifier : a new configuration", IEE proceedings, Vol :136, No : 2 (1989), pp : 57-61

[9] Feng-Tso. Chein, Yi-Jen. Chan, "Bandwidth enhancement of transimpedance amplifier by a capacitive-peaking design", IEEE Journal of Solid-State Circuits, Vol : 34, No : 8 (1999), $p p: 1167-1170$

[10] C. Seidl, J.Knorr , H. Zimmermann, "Simple feedback network for bandwidth enhancement of transimpedance amplifiers", Electronics Letters, Vol : 39, No : 25 (2003)

[11] Hoyle, Peyton," Bootstrapping Techniques to improve the bandwidth of transimpedance amplifiers" Analog Signal processing, IEE Colloquium, 1998. 
[12] Centurelli, F. Luzzi, R. Olivieri, M. Trifiletti, A, “ A Bootstrap Technique for Wideband Amplifiers" IEEE Transaction on Circuit and Systems, Vol 49, No. 10, 2002.

[13] Idrus, S. M, Rais, S. S, Ramli, A," Bandwidth Analysis of Bootstrap Transimpedance Amplifier for Optical Free Space Receiver, ELEKTRIKA, Vol 10, No. 1, pp 13-19, 2008

[14] Idrus, S. M, Ngajikin, N. H, Nik Abd Malik, N. N, Aziz, S.I.A, "Performance Analysis of Bootstrap Transimpedance Amplifier for Large Window Optical Wireless Receiver" 2006 International RF and Microwave Conference Proceedings.

[15] Abdullah M.F.L,Green.R, Leeson.M, "Optical wireless communication front-ends" High frequency Postgraduate Student Colloquium 2004, pp : 3-8

[16] Abdullah M.F.L, Green R.J, "Receiver Amplifier Technique for Dynamic Service Quality Adaptation In Optical Wireless Communication" $4^{\text {th }}$ International Symposium on Broadband Communication (ISBC 2010), 11-14 July 2010.

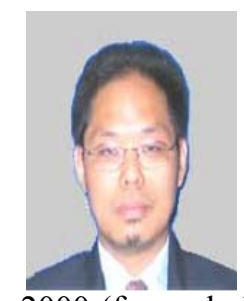

Mohammad Faiz Liew Abdullah received his BSc (Hons) in Electrical Engineering (Communication) in 1997, Dip Education in 1999 and Meng (Research) in the area of Optical Fiber Communication in 2000 from University of Technology Malaysia (UTM). He completed his PhD in August 2007 from The University of Warwick, United Kingdom in Wireless Optical Communication Engineering. He started his career as a lecturer at Polytechnic Seberang Prai (PSP) in 1999 and was transferred to UTHM in 2000 (formerly known as PLSP). At present he is an Associate Professor in the Department of Communication Engineering, Faculty of Electrical \& Electronic Engineering, University Tun Hussein Onn Malaysia (UTHM). He had more than 10 years of experience in teaching higher education, which involved the subjects of Optical Fiber Communication, Advanced Optical Communication, Advanced Digital Signal Processing and etc. His research area of interest are Wireless and Optical Communication, WiMAX, Robotic with Communication. 